\title{
PRIMEROS REGISTROS PARA CHILE DE DOS ESPECIES DE PILOBALIA BURMEISTER, 1875 (COLEOPTERA, TENEBRIONIDAE)
}

\author{
FIRST RECORDS FOR CHILE OF THE TWO SPECIES OF PILOBALIA \\ BURMEISTER, 1875 (COLEOPTERA, TENEBRIONIDAE)
}

\begin{abstract}
In the present work, the first Chilean records of Pilobalia torresi Molinari, 1968 and Pilobalia escobari Peña, 1973 (Coleoptera: Tenebrionidae: Nycteliini) are reported. The specimens were collected by hand in Colchane $\left(19^{\circ} 16^{\circ}\right.$ $\left.60^{\prime \prime} \mathrm{S}, 68^{\circ} 37^{\prime} 60^{\prime \prime} \mathrm{W}\right)$, in the Altiplano of the Region of Tarapacá, Chile. A redescription of the species and an environmental characterization of the collection sites are provided.
\end{abstract}

\section{Marcos A. Ferrú ${ }^{1} \&$ Jaime Pizarro-Araya ${ }^{2}$}

${ }^{1}$ J. J. Pérez \# 429, San Bernardo, Santiago, Chile. Email: m_ferru@hotmail.com ${ }^{2}$ Laboratorio de Entomología Ecológica, Departamento de Biología, Facultad de Ciencias, Universidad de La Serena; Casilla 599, La Serena, Chile. Email: japizarro@userena.cl

La tribu Nycteliini, de la subfamilia Pimeliinae (Watt 1974, Doyen 1993, Bouchard et al. 2005), incluye 12 géneros y 306 especies, distribuidas en regiones áridas y semiáridas de Argentina, Bolivia, Brasil, Chile, Paraguay y Perú (Flores \& Triplehorn 2002). En Chile se ha informado de la presencia de 8 géneros: Nyctelia Latreille, 1825 (15 especies en Chile), Gyriosomus GuérinMéneville, 1834 (38), Epipedonota Solier, 1836 (5), Callyntra Solier, 1836 (17), Auladera Solier, 1836 (4), Mitragenius Solier, 1836 (3), Psectrascelis Solier, 1836 (36) y Pilobalia Burmeister, 1875 (3).

Pilobalia, del cual se han descrito 46 especies hasta la fecha, es uno de los géneros más diversificados de Nycteliini (Kulzer 1954, Flores 1997, Flores \& Pizarro-Araya 2006), y está representado en Chile por Pilobalia decorata immaculata (Blanchard, 1847), Pilobalia oblonga (Blanchard, 1847) y Pilobalia elegans (Blanchard, 1847), todas distribuidas en sectores altoandinos de la I y II regiones del país (Peña 1966, Flores 1997). El objetivo del presente trabajo es dar a conocer la presencia en Chile de otras dos especies de Pilobalia
Los ejemplares fueron capturados en la localidad altiplánica de Colchane, en la provincia de Iquique, I Región de Tarapacá (Fig. 1). La determinación taxonómica de los ejemplares se basó en la clasificación de Kulzer (1954) y Peña (1973). Los ejemplares estudiados se encuentran depositados en la sección de Entomología del Museo Nacional de Historia Natural (MNNC), Santiago, Chile.

\section{Pilobalia torresi Molinari, 1968}

(Fig. 2A)

Localidad tíPICA: ARGENTINA; Provincia de Jujuy; Departamento de Humahuaca; Iturbe $\left(22^{\circ}\right.$ 57' 36' S, 65'22' 48', O) (Fig. 1).

Material examinado: CHILE; I Región de Tarapacá; Provincia de Iquique; Colchane; 3 hembras; 10-03-2001; coll. M. Ferrú; MNNC (Fig. 1).

DESCRIPCIÓN MORFOLÓGICA: especie de color negro brillante, longitud corporal de $15 \mathrm{~mm}$ (rango: 15$16 \mathrm{~mm}$ ). Cabeza: gruesamente punteada y con cerditas negras; antena con el tercer segmento más 
largo que los restantes. Tórax: pronoto el doble de ancho que su largo, ancho mayor en el tercio posterior, margen posterior con ángulos casi rectos; con puntación escasa y débil; con una débil impresión junto al margen de la parte más ancha; ornado lateral y superiormente con cerditas cortas y negras; ángulos anteriores obtusos, redondeados y fuertemente curvados. Elitros: glabros con franja aterciopelada de color amarillento en el extremo distal; la base, que es tan ancha como la del protórax, se ensancha gradualmente hasta el tercio posterior y de ahí se angosta casi en línea recta hasta los ápices.
Patas: tibias con densos pelos blancos (Fig. 2A).

\section{Pilobalia escobari Peña, 1973}

(Fig. 2B)

LocAlidAd TíPICA: ARGENTINA; Provincia de Jujuy; Departamento de Yavi; Pumahuasi $\left(22^{\circ} 17^{\prime} 24^{\prime \prime} \mathrm{S}\right.$, $65^{\circ} 40^{\prime} 12^{\prime \prime}$ O) (Fig. 1).

Material eXaminado: CHILE; I Región de Tarapacá; Provincia de Iquique; Colchane; 1 hembra, 1 macho; 10-03-2001; coll. M. Ferrú; MNNC (Fig. 1).

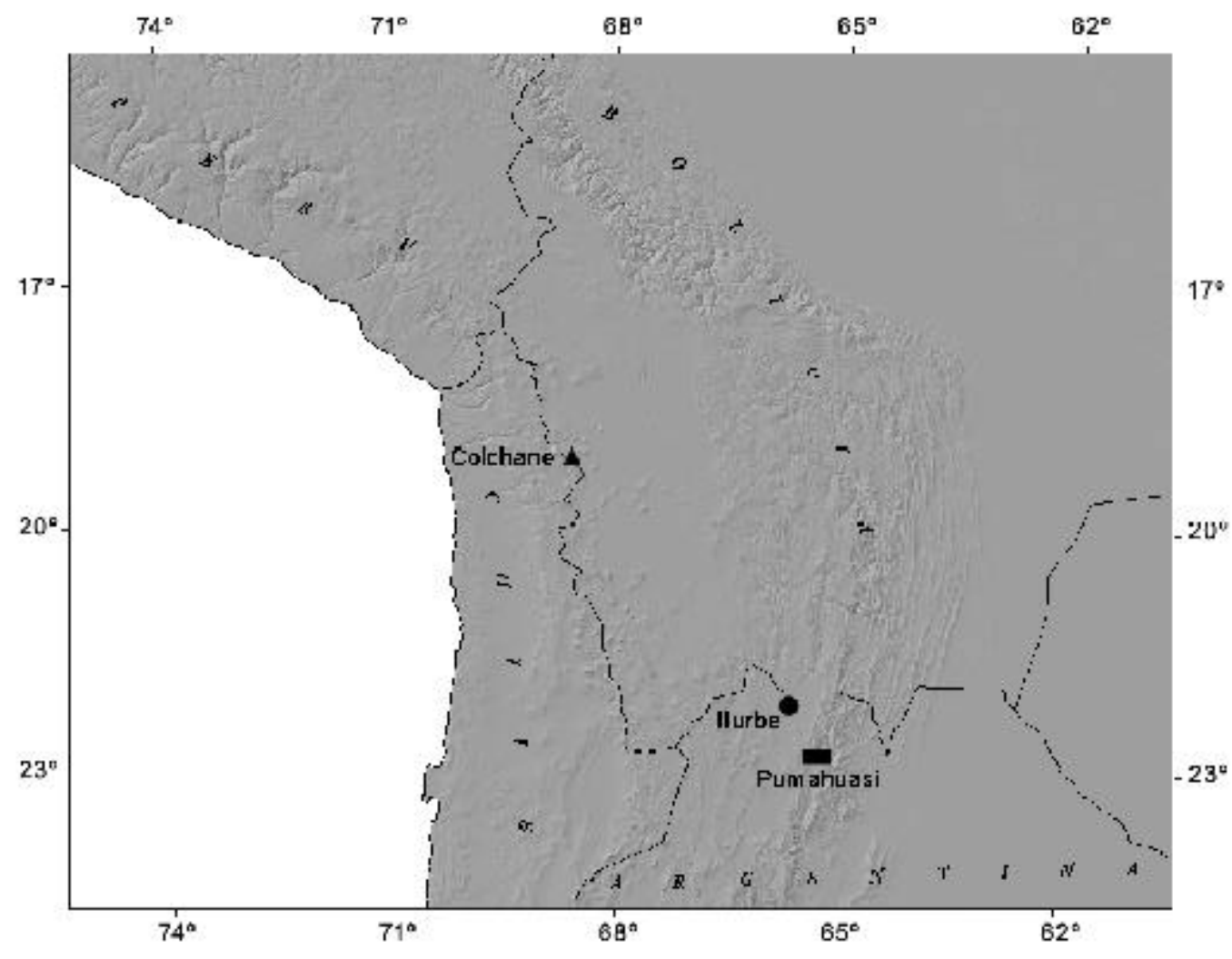

Figura 1. Localidad típica de Pilobalia torresi ( • ) y Pilobalia escobari ( $\boldsymbol{\square}$ ), y localidad de registro de ambas especies en Chile $(\boldsymbol{\Lambda})$.

FIGURE 1. Typical locality of Pilobalia torresi

( ) and Pilobalia escobari

$i(\boldsymbol{\square})$, and locality in Chile where both species were recorded $(\boldsymbol{\Delta})$. 
DESCRIPCIÓN MORFOLÓGICA: color negro brillante, longitud corporal de $20 \mathrm{~mm}$ (rango: 19-20 mm). Cabeza: con pelos negros y aislados en la frente; en el borde de los ojos presenta pelos amarillos que forman una franja que los rodea (estos pelos también se encuentran más dispersos en la sutura clipeal y en el borde del clípeo); clípeo profundo y ancho en su parte media, que se angosta hacia los lados formando un pliegue; segundo y tercer segmento antenal en con- junto con longitud mayor que la suma del cuarto y del quinto. Tórax: ancho de la base del protórax mayor que el lado más ancho de los élitros. Elitros: franja de pelos sedosos que abarcan desde la base de los élitros hasta el extremo distal, a través del borde externo de los élitros; dicha banda no entra en contacto con las costillas; además, hay reminiscencias de este tomento en la zona media de los élitros, donde aparecen algunos pelos canos (Fig. 2B).
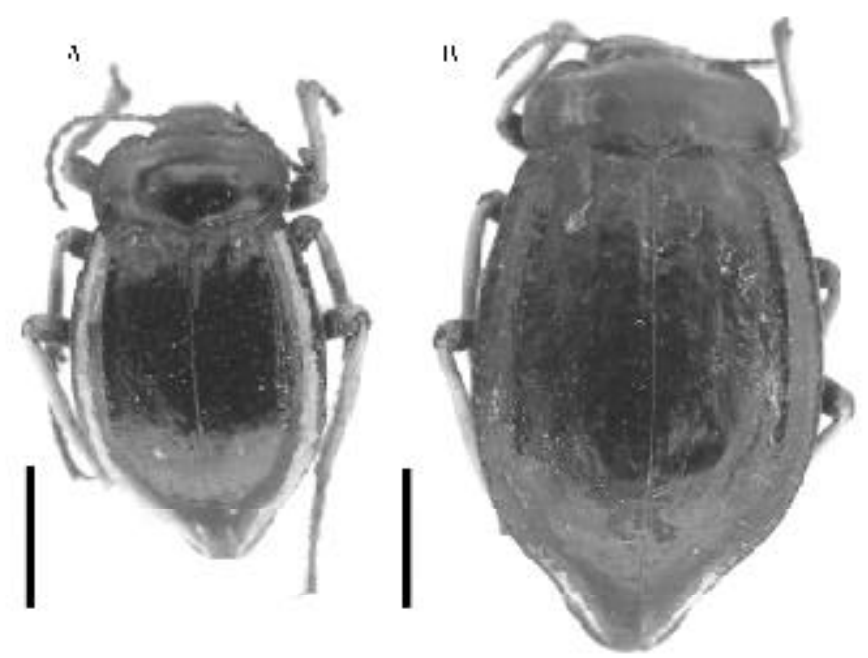

FIgUra 2. A. Vista dorsal de Pilobalia torresi, escala $=5 \mathrm{~mm}$. B. Vista dorsal de Pilobalia escobari, escala = $5 \mathrm{~mm}$.

Figure 2. A. Dorsal view of Pilobalia torresi, scale $=5 \mathrm{~mm}$. B Dorsal view of Pilobalia escobari, scale $=5$ $\mathrm{mm}$.

HÁBITAT: Es posible insertar a la localidad de Colchane en la formación vegetacional. Estepa Altoandina Subdesértica (Gajardo 1995). La vegetación característica de esta franja corresponde a Festuca chrysophylla, Stipa chrysophylla, Nassella nardoides (Poaceae), Parastrephiaquadrangularis (Asteraceae), Adesmia spinosissima (Fabaceae) y Senecio aff. puchii (Asteraceae). En los sectores rocosos abundan los cojines de Azorella compacta (Apiaceae), Pycnophyllum bryoides, Pycnophyllum molle (Caryophyllaceae), Junellia tridactila (Verbenaceae). En los arenales son frecuentes las hierbas en roseta, como Astragalus cryptanthus (Fabaceae), Moschopsis monocephala (Calyceraceae) y Phacelia setigera (Hydro- phyllaceae) (Villagrán et al. 2003). Los suelos son de tipo arenoso-rocoso y presentan vegetación de bajo tamaño muy dispersa (Villagrán et al. 2003). Puesto que el clima es tropical de altura, las lluvias de verano producen la floración durante marzoabril; la temperatura promedio mensual es de $-6,49$ ${ }^{\circ} \mathrm{C}$, siendo más baja de $-22,95{ }^{\circ} \mathrm{C}$ en el mes de septiembre, y la temperatura media mensual es de 5,11 ${ }^{\circ} \mathrm{C}$, siendo la mayor de $24,15{ }^{\circ} \mathrm{C}$ en el mes de enero. Estos datos corresponden a los obtenidos por la estación meteorológica del Salar de Coposa (2040’45', S, 6842'27' 'O), de la Compañía Minera Doña Inés de Collahuasi, ubicada a $170 \mathrm{~km}$ del lugar donde se recolectaronlos ejemplares aquí estudiados. 
Debido a estos nuevos registros, las especies de Pilobalia conocidas en Chile llegan a cinco. Es probable que muestreos más exhaustivos, en sectores de difícil acceso, permitan ampliar los rangos distribucionales de éstas y también de otras especies de Pilobalia. Al respecto, Jerez (2000) señala que la mayoría de la información disponible, tanto en colecciones y literatura, corresponde a recolecciones efectuadas cerca de poblados o a la red vial, originando en conjunto una información local incompleta. En este sentido, muestreos en la Reserva Nacional Las Vicuñas y en el Monumento Natural Salar de Surire, pueden ser de relevancia para el inventario general y categorización de futuros estados de conservación de la fauna de artrópodos de altura, lo que permitiría contribuir a un manejo de esta área silvestre.

\section{AGRADECIMIENTOS}

A Gustavo E. Flores (IADIZA-CRICYT, Argentina), Mario Elgueta (MNNC, Chile), y a un revisor anónimo por los aportes realizados al manuscrito. A David López Aspe (CEAZA, Chile) por la asistencia en SIG. A la Compañía Minera Doña Inés de Collahuasi, por la información meteorológica, y a la Dirección de Investigación de la Universidad de La Serena.

\section{BIBLIOGRAFIA}

Bouchard, P., J.F. Lawrence, A.E. Davies \& A.F. Newton. 2005. Synoptic classification of the world Tenebrionidae (Insecta: Coleoptera) with a review of family-group names. Annales Zoologici 55:499-530.

Burmeister, H. 1875. Melanosoma Argentina. Stettiner Entomologische Zeitung 36:457-500.
Doyen, J.T. 1993. Cladistic relationships among Pimeliinae Tenebrionidae (Coleoptera). Journal of the New York Entomological Society 101:443514.

Flores, G.E. 1997. Revisión de la Tribu Nycteliini (Coleoptera: Tenebrionidae). Revista de la Sociedad Entomológica Argentina 56:1-19.

Flores, G.E. \& C.A. Triplehorn. 2002. Entomobalia new genus, the first member of Nycteliini (Coleoptera: Tenebrionidae) from Brazil. Proceedings of the Entomological Society of Washington 104:602613.

Flores, G.E. \& J. Pzarro-Araya. 2006. The Andes mountain range as a vicariant event in the Pimeliinae (Coleoptera: Tenebrionidae) in southern South America. Cahiers Scientifiques 10:95-102.

Gajardo, R. 1993. La vegetación natural de Chile. Editorial Universitaria, Santiago, Chile. 165 pp.

JEREZ, V. 2000. Diversidad y patrones de distribución geográfica de insectos coleópteros en ecosistemas desérticos de la Región de Antofagasta, Chile. Revista Chilena de Historia Natural 73:79-92.

Kulzer, H. 1954. Neunter Beitrag zur Kenntnis der Tenebrioniden (Col.) Eine Studie über die Tribus Nycteliini. Entomologische Arbeiten aus dem Museum George Frey 5:14-267.

Molinari, H. 1968. Tenebriónidos nuevos de Jujuy (República Argentina) (Coleoptera-Tenebrionidae). Revista de la Sociedad Entomológica Argentina XXXI:133-140.

Peña, L.E. 1966. Catálogo de los Tenebrionidae (Coleoptera) de Chile. Entomologische Arbeiten aus dem Museum George Frey 17:397-453.

Peña, L.E. 1973. Insectos de la zona Altiplánica de la Argentina. II. El género Pilobalia Burmeister (Coleoptera, Tenebrionidae). Revista de la Sociedad Entomológica Argentina 34:161-176.

Villagrán, C., M. R omo \& V. CAStro. 2003. Etnobotánica del sur de los Andes de la Primera Región de Chile: un enlace entre las Culturas Altiplánicas y las de Quebradas Altas del Loa Superior. Región de Chile. Chungará (Arica) 35:73-124.

WATT, J.C. 1974. A revised subfamily classification of Tenebrionidae (Coleoptera). New Zealand Journal of Zoology 1:381-452. 